\title{
Evaluation of the impact of laboratory accreditation on downstream outcomes
}

\author{
Daniel Shpilsky, MD, a and Matthew E. Harinstein, MD, MBA, FACC ${ }^{a}$ \\ a Heart and Vascular Institute, University of Pittsburgh Medical Center, Pittsburgh, PA
}

Received Jul 13, 2020; accepted Jul 13, 2020

doi: $10.1007 / \mathrm{s} 12350-020-02292-0$

\section{See related article, pp. 2952-2961}

Noninvasive assessment of myocardial ischemia, with stress echocardiography and myocardial perfusion imaging, is a key component of the evaluation of cardiac symptoms and risk stratification. Multiple organizations have developed joint consensus appropriate use criteria for the use of echocardiography, myocardial perfusion imaging (MPI), and assessment of ischemic heart disease. $^{1-3}$ These stress studies are performed in both accredited and non-accredited laboratories; however, the impact of that is not well known. Since 2007, the accreditation of laboratories performing noninvasive cardiac testing has become more prevalent and accreditation is often mandated by Medicare as well as private, third-party insurers. ${ }^{4}$ The organizations responsible for providing accreditation include the Intersocietal Accreditation Commission (IAC) and the American College of Radiology (ACR) which oversee noninvasive cardiovascular imaging laboratories including echocardiography, nuclear medicine, nuclear cardiology, positron emission tomography, magnetic resonance imaging, and computed tomography. The intended goal of accreditation is to demonstrate a national standard of quality and competency from laboratories performing cardiac imaging regardless of where the study is performed or interpreted. The achievement of these standards is expected to most importantly improve patient clinical outcomes and to guide further care and

Reprint requests: Matthew E. Harinstein, MD, MBA, FACC, Heart and Vascular Institute, University of Pittsburgh Medical Center, South Tower 3F, E352.2, 200 Lothrop Street, Pittsburgh, PA 15213; harinsteinme@upmc.edu

J Nucl Cardiol 2021;28:2962-4.

$1071-3581 / \$ 34.00$

Copyright (c) 2020 American Society of Nuclear Cardiology. decision-making. It may also have the associated benefit of decreasing downstream extraneous testing and unnecessary procedures. Evidence supporting these claims has mostly been limited to facility-reported surveys. Some of the purported benefits of laboratory accreditation include a perceived improvement in laboratory operations and standardization, staff knowledge, image quality, guideline adherence, and report completeness. $^{5-7}$ Several societies including the American College of Cardiology (ACC), American Society of Nuclear Cardiology (ASNC), and the American Society of Echocardiography (ASE) have instituted recommendations and even mandates regarding the accreditation of laboratories. Despite the potential benefits, a universal mandate to achieve accreditation does not yet exist and many imaging laboratories remain unaccredited. This lack of universal accreditation is likely due to significant challenges that facilities perceive about the process. These include the length of time needed to prepare appropriate documents for the accreditation process, the need for repeat 3-year reaccreditation, the length of time to receive the final accreditation decision, the need for continuing medical education in a specific diagnostic testing modality, the lack of simplicity in reaccreditation, and the cost of accreditation (especially for multiple modality laboratories). ${ }^{4}$

Prior studies have evaluated the significance of accreditation status on imaging quality, report completeness, and clinical outcomes. In a study by Thaden et al, accredited echocardiography laboratories had more complete reporting and better imaging quality when evaluating patients with valvular heart disease. ${ }^{8}$ It has also been demonstrated that accreditation of vascular laboratories was associated with more accurate characterization of carotid artery stenosis. However, this was not associated with improved clinical outcomes in patients who underwent carotid endarterectomy. ${ }^{9}$ The impact of accreditation on clinical outcomes in patients undergoing stress testing for the evaluation of ischemic heart disease has not been well described in the 
literature. Specifically, the association of accreditation with subsequent cardiac testing, downstream healthcarerelated costs, or clinical outcomes has not been previously evaluated.

In this issue of the Journal of Nuclear Cardiology, Shah et al. report their findings on subsequent testing and clinical outcomes in patients undergoing index stress echocardiogram or MPI tests in accredited vs non-accredited facilities. ${ }^{10}$ In their analysis, they evaluated 20 stress echocardiogram facilities (7 accredited) and $37 \mathrm{MPI}$ facilities (11 accredited) in Maine from 2012 to 2014. A total of 4603 stress echocardiograms and 8449 MPI studies were considered in the analysis. CPT and ICD-9 codes were used to identify patients who received additional diagnostic testing within six months of the index test in addition to identifying clinical events (i.e., cardiac catheterization, revascularization with PCI or CABG, and cardiac outcomes such as hospitalization for angina, unstable angina, acute myocardial infarction, cardiac arrest, and heart failure). Multivariate analysis showed higher odds of subsequent MPI testing and hospitalization for angina if the index test was performed in a non-accredited laboratory in both the stress echocardiogram and MPI cohorts. The authors also found a higher likelihood of subsequent PCI (OR 1.68, (95\% CI 1.13-2.50)), if the initial MPI study was done in a non-accredited laboratory. Thus, the authors conclude that cardiac testing completed in non-accredited facilities is associated with higher odds of subsequent MPI testing, hospitalization for angina, and PCI. The authors postulate that this additional testing may result in delayed diagnosis, increased exposure to radiation, and higher healthcare delivery costs.

This study expands on the existing literature evaluating the benefits of noninvasive cardiovascular testing performed at accredited laboratories which has previously found improvement in image quality and more complete study reporting, among potential additional benefits. Specifically, this study suggests that index stress echocardiograms or MPI tests performed at accredited facilities may decrease superfluous downstream testing and result in improved patient outcomes. Laboratory accreditation generally demonstrates a commitment to excellence, but previous work has shown that it is important to maintain that commitment. ${ }^{11,12}$ In a study evaluating both echocardiography and nuclear cardiology laboratory accreditation applications, factors such as maintenance and utilization of contemporary equipment were found to be associated with laboratory quality metrics with a trend toward lower quality with older equipment. ${ }^{13}$ Thus, variations in outcomes may exist even within the accredited laboratory group based on differences in experience, volumes, and equipment. One discrepancy in the authors' outcomes was the higher rates of acute myocardial infarction and hospitalization for heart failure in patients undergoing testing at accredited facilities. The authors postulate that a combination of factors may be present in patients undergoing testing at accredited facilities including a higher comorbidity index, a higher percentage of patients with prior or known coronary artery disease, increased vascular inflammation, and plaque composition that is more prone to adverse events.

There are limitations of the study which should be noted. First, this is a retrospective review of CPT/ICD-9 codes from medical claims, which do not allow for analysis of diagnostic testing appropriateness, evaluation of image quality, or interpretation of the final report. Additionally, it should be noted that this study was performed in a single state comprised of mostly rural counties. As noted, this population has a lower uninsured rate compared to the national average and given the mostly rural population has potentially older patients, more poverty, and lower median income which may make these findings less applicable to a more demographically diverse patient population. Thus, further research in areas with different demographics would be warranted. Additionally, a more rural area may have fewer healthcare providers and accredited laboratories in close proximity thus limiting the ability or choice to have a study performed in a particular facility.

Shah et al. performed an important study to better understand the value of accreditation. They evaluated the two most conventional imaging methods for stress testing in the assessment of ischemic heart disease. They made insightful observations regarding further downstream testing as well as the utilization of invasive procedures in these patients. However, whether these procedures improved clinical outcomes or were appropriate based on appropriateness criteria is unknown. In a previously performed meta-analysis evaluating outcomes after inappropriate MPI tests, Elgendy et al. observed that inappropriate MPI studies are less likely to be abnormal or reveal evidence of myocardial ischemia. ${ }^{14}$ Thus, inappropriate studies may be less likely to alter outcome and may result in unnecessary expenditures. This highlights the need to take the next step to evaluate the clinical outcomes of patients who underwent these studies. In order to determine whether echocardiography and nuclear cardiology lab accreditation are truly associated with improved clinical outcomes and reduced downstream healthcare costs, more studies examining the downstream effects on subsequent testing, utilization of invasive procedures, and patient-centered clinical outcomes are needed. If future studies do find significant benefit, it would be an important step forward in the further promotion and potential universal mandating of laboratory accreditation. 


\section{Disclosures}

Daniel Shpilsky and Matthew E. Harinstein have no disclosures.

\section{References}

1. Doherty JU, Kort S, Mehran R, Schoenhagen P, Soman P, Dehmer GJ, et al. ACC/AATS/AHA/ASE/ASNC/HRS/SCAI/SCCT/ SCMR/STS 2019 appropriate use criteria for multimodality imaging in the assessment of cardiac structure and function in nonvalvular heart disease: A report of the American College of Cardiology Appropriate Use Criteria Task Force, American Association for Thoracic Surgery, American Heart Association, American Society of Echocardiography, American Society of Nuclear Cardiology, Heart Rhythm Society, Society for Cardiovascular Angiography and Interventions, Society of Cardiovascular Computed Tomography, Society for Cardiovascular Magnetic Resonance, and the Society of Thoracic Surgeons. J Am Coll Cardiol. 2019;73(4):488-516.

2. Douglas PS, Garcia MJ, Haines DE, Lai WW, Manning WJ, Patel AR, et al. ACCF/ASE/AHA/ASNC/HFSA/HRS/SCAI/SCCM/ SCCT/SCMR 2011 Appropriate use criteria for echocardiography. A report of the American College of Cardiology Foundation Appropriate Use Criteria Task Force, American Society of Echocardiography, American Heart Association, American Society of Nuclear Cardiology, Heart Failure Society of America, Heart Rhythm Society, Society for Cardiovascular Angiography and Interventions, Society of Critical Care Medicine, Society of Cardiovascular Computed Tomography, Society for Cardiovascular Magnetic Resonance American College of Chest Physicians. J Am Soc Echocardiogr. 2011;24(3):229-67.

3. Ronan G, Wolk MJ, Bailey SR, Doherty JU, Douglas PS, Hendel $\mathrm{RC}$, et al. ACCF/AHA/ASE/ASNC/HFSA/HRS/SCAI/SCCT/ SCMR/STS 2013 multimodality appropriate use criteria for the detection and risk assessment of stable ischemic heart disease: A report of the American College of Cardiology Foundation Appropriate Use Criteria Task Force, American Heart Association, American Society of Echocardiography, American Society of Nuclear Cardiology, Heart Failure Society of America, Heart Rhythm Society, Society for Cardiovascular Angiography and Interventions, Society of Cardiovascular Computed Tomography,
Society for Cardiovascular Magnetic Resonance, and Society of Thoracic Surgeons. J Nucl Cardiol. 2014;21(1):192-220.

4. Heller GV, Katanick SL, Sloper T, Garcia M. Accreditation for cardiovascular imaging: Setting quality standards for patient care. JACC Cardiovasc Imaging. 2008;1(3):390-7.

5. Jerome SD, Farrell MB, Godiwala T, Heller GV, Bezold LI, Choi JY, et al. Facility perception of nuclear cardiology accreditation: Results of an Intersocietal Accreditation Commission (IAC) survey. J Nucl Cardiol. 2015;22(3):496-503.

6. Maddux PT, Farrell MB, Ewing JA, Tilkemeier PL. Improved compliance with reporting standards: A retrospective analysis of Intersocietal Accreditation Commission Nuclear Cardiology Laboratories. J Nucl Cardiol. 2018;25(3):986-94.

7. Manning WJ, Farrell MB, Bezold LI, Choi JY, Cockroft KM, Gornik HL, et al. How do noninvasive imaging facilities perceive the accreditation process? Results of an Intersocietal Accreditation Commission Survey. Clin Cardiol. 2015;38(7):401-6.

8. Thaden JJ, Tsang MY, Ayoub C, Padang R, Nkomo VT, Tucker $\mathrm{SF}$, et al. Association between echocardiography laboratory accreditation and the quality of imaging and reporting for valvular heart disease. Circ Cardiovasc Imaging. 2017;10(8):e006140.

9. Brown OW, Bendick PJ, Bove PG, Long GW, Cornelius P, Zelenock GB, et al. Reliability of extracranial carotid artery duplex ultrasound scanning: Value of vascular laboratory accreditation. J Vasc Surg. 2004;39(2):366-71.

10. Shah JN MK, Lucas FL, Fairfield KM, Cohen MC. Variation in additional testing and patient outcomes after stress echocardiography or myocardial perfusion imaging, according to accreditation status of testing site. J Nucl Cardiol. 2020; [in press].

11. What is IAC Echocardiography Accreditation? https://www.inte rsocietal.org/echo/main/what_is_accreditation.htm.

12. What is IAC Nuclear/PET Accreditation? https://www.intersocieta 1.org/nuclear/main/what_is_accreditation.htm.

13. Malhotra S, Farrell MB, Katz WE, Soman P. The association of imaging equipment age with other quality metrics and successful laboratory accreditation by the Intersocietal Accreditation Commission. Echocardiography. 2019;36(9):1615-24.

14. Elgendy IY, Mahmoud A, Shuster JJ, Doukky R, Winchester DE. Outcomes after inappropriate nuclear myocardial perfusion imaging: A meta-analysis. J Nucl Cardiol. 2016;23(4):680-9.

Publisher's Note Springer Nature remains neutral with regard to jurisdictional claims in published maps and institutional affiliations. 